\title{
A note on orthogonal similitude groups
}

\author{
C. RYAN VINROOT* \\ Department of Mathematics, Tata Institute of Fundamental Research, \\ Homi Bhabha Road, Mumbai - 400 005, India \\ Communicated by R. Guralnick
}

(Received 18 October 2004; in final form 24 March 2005)

\begin{abstract}
Let $V$ be a vector space over the field $F$ such that $\operatorname{char}(F) \neq 2$, and let $V$ have a symmetric nondegenerate bilinear form. Let $\mathrm{GO}(V)$ be the orthogonal similitude group for this symmetric form, with similitude character $\mu$. We prove that if $g \in \mathrm{GO}(V)$ with $\mu(g)=\beta$, then $g=t_{1} t_{2}$ where $t_{1}$ is an orthogonal involution, and $t_{2}$ is such that $t_{2}^{2}=\beta I$ and $\mu\left(t_{2}\right)=\beta$. As an application, we obtain an expression for the sum of the degrees of the irreducible characters of $\mathrm{GO}\left(n, \mathbb{F}_{q}\right)$ for odd $q$.
\end{abstract}

Keywords: Similitude group; Orthogonal group; Factorization of matrices; Characters of the similitude group over a finite field

2000 AMS Subject Classifications: 15A23; 20G40

\section{Introduction}

This note is an addendum to [1], where we obtain a factorization in the symplectic similitude group. In Theorem 1 below, we obtain a factorization in the group of orthogonal similitudes $\mathrm{GO}(V)$, where $V$ is an $F$-vector space with $\operatorname{char}(F) \neq 2$, and the similitude character is $\mu$. The method is the same as in [1], and the notation established there is freely used. In the proof of Theorem 1, we refer to [1] to all parts that immediately apply to the orthogonal case, while any changes that are needed in the proof are given specifically.

As an application of Theorem 1, we use a result of Gow [2] on the orthogonal group over a finite field to obtain information on the characters of the orthogonal similitude group over a finite field, as given in Theorem 2 and Corollary 2. In a paper to appear by Adler and Prasad [3], Corollary 1 is used to prove a theorem on $p$-adic groups. In particular, if $V$ is a vector space over a $p$-adic field, Adler and Prasad prove that any irreducible admissible representation of $\mathrm{GO}(V)$ restricted to $\mathrm{O}(V)$ is multiplicity free, and they also prove the corresponding statement for the symplectic similitude group.

*Email: vinroot@math.tifr.res.in 


\section{The main theorem}

Let $V$ be an $F$-vector space, with $\operatorname{char}(F) \neq 2$, equipped with a nondegenerate bilinear symmetric form $\langle\cdot, \cdot\rangle: V \times V \rightarrow F$. Then the orthogonal group of similitudes of $V$ with respect to this form is the group $\mathrm{GO}(V)=\{g \in \mathrm{GL}(V):\langle g v, g w\rangle=\mu(g)\langle v, w\rangle$ for some $\mu(g) \in F^{\times}$for all $\left.v, w \in V\right\}$. Then $\mu: \mathrm{GO}(V) \rightarrow F^{\times}$is a multiplicative character called the similitude character, and the orthogonal group is $\mathrm{O}(V)=\operatorname{ker}(\mu)$.

THEOREM 1 Let $g$ be an element of $\mathrm{GO}(V)$ satisfying $\mu(g)=\beta$. Then we may factor $g$ as $g=t_{1} t_{2}$, where $t_{1}$ is an orthogonal involution and $t_{2}$ satisfies $t_{2}^{2}=\beta I$ and $\mu\left(t_{2}\right)=\beta$.

Proof Wonenburger [4] proved that any element of $\mathrm{O}(V)$ is the product of two orthogonal involutions. So if $\mu(g)=\beta$ is a square in $F$, then the theorem follows directly from Wonenburger's result. So we assume $\beta$ is not a square.

As in [1], for any monic polynomial $f \in F[x]$ of degree $d$, define the $\beta$-adjoint of $f$ to be

$$
\hat{f}(x)=f(0)^{-1} x^{d} f(\beta / x)
$$

and define a monic polynomial to be self- $\beta$-adjoint if $\hat{f}=f$. Then, for any $g \in \operatorname{GO}(V)$, the minimal polynomial of $g$ is self- $\beta$-adjoint. All of the results in sections 2 and 3 of [1] are valid for transformations $g$ which are self- $\beta$-adjoint, as the proofs only use this fact. These results reduce us to looking at the case that either $g$ is a cyclic transformation for $V$, that is $V$ is generated by vectors of the form $g^{i} v$ for some $v \in V$, or the case that $g$ has minimal polynomial of the form $q(x)^{s}$, where $q(x)$ is an irreducible self- $\beta$-adjoint polynomial.

We deal with the cyclic case first. In [1, Proposition 3(i)], we prove that if $g \in \operatorname{GL}(V)$ is a cyclic transformation with self- $\beta$-adjoint minimal polynomial, ignoring any inner product structure, then we can factor $g=t_{1} t_{2}$ such that $t_{1}^{2}=I$ and $t_{2}^{2}=\beta I$. This is proven as follows. If $V$ is cyclic for the vector $v$, then we let $P$ be the space spanned by vectors of the form $\left(g^{i}+\beta^{i} g^{i}\right) v$ and let $Q$ be the space spanned by the vectors of the form $\left(g^{i}-\beta^{i} g^{i}\right) v$. Then $V=P \oplus Q$, and the transformation having $P$ as its +1 eigenspace and $Q$ as its -1 eigenspace is exactly the involution $t_{1}$ that we seek. For the case that $g \in \mathrm{GO}(V)$, we must show that this $t_{1}$ is orthogonal. Let $\left(g^{i}+\beta^{i} g^{i}\right) v \in P$ and $\left(g^{j}-\beta^{j} g^{j}\right) v \in Q$. Then we have:

$$
\begin{aligned}
& \left\langle\left(g^{i}+\beta^{i} g^{-i}\right) v,\left(g^{j}-\beta^{j} g^{-j}\right) v\right\rangle \\
& =\left\langle\left(g^{i}+\beta^{i} g^{-i}\right) v, g^{j} v\right\rangle-\left\langle g^{i} v, \beta^{j} g^{-j} v\right\rangle-\left\langle\beta^{i} g^{-i} v, \beta^{j} g^{-j} v\right\rangle \\
& =\left\langle\left(g^{i}+\beta^{i} g^{-i}\right) v, g^{j} v\right\rangle-\left\langle g^{j} v, \beta^{i} g^{-i} v\right\rangle-\left\langle g^{j} v, g^{i} v\right\rangle \\
& =\left\langle\left(g^{i}+\beta^{i} g^{-i}\right) v, g^{j} v\right\rangle-\left\langle g^{j} v,\left(g^{i}+\beta^{i} g^{-i}\right) v\right\rangle \\
& =0 .
\end{aligned}
$$

So $P$ and $Q$ are mutually orthogonal. Now let $u$ and $u^{\prime}$ be any two vectors in $V=P \oplus Q$. Write $u=w+y, u^{\prime}=w^{\prime}+y^{\prime}$, where $w, w^{\prime} \in P$ and $y, y^{\prime} \in Q$. We compute $\left\langle t_{1} u, t_{1} u^{\prime}\right\rangle$ :

$$
\begin{aligned}
\left\langle t_{1} u, t_{1} u^{\prime}\right\rangle & =\left\langle t_{1}(w+y), t_{1}\left(w^{\prime}+y^{\prime}\right)\right\rangle=\left\langle w-y, w^{\prime}-y^{\prime}\right\rangle \\
& =\left\langle w, w^{\prime}\right\rangle+\left\langle y, y^{\prime}\right\rangle-\left\langle y, w^{\prime}\right\rangle-\left\langle w, y^{\prime}\right\rangle \\
& =\left\langle w, w^{\prime}\right\rangle+\left\langle y, y^{\prime}\right\rangle .
\end{aligned}
$$


While computing $\left\langle u, u^{\prime}\right\rangle$ gives us:

$$
\begin{aligned}
\left\langle u, u^{\prime}\right\rangle & =\left\langle w+y, w^{\prime}+y^{\prime}\right\rangle \\
& =\left\langle w, w^{\prime}\right\rangle+\left\langle y, y^{\prime}\right\rangle+\left\langle y, w^{\prime}\right\rangle+\left\langle w, y^{\prime}\right\rangle \\
& =\left\langle w, w^{\prime}\right\rangle+\left\langle y, y^{\prime}\right\rangle .
\end{aligned}
$$

Therefore, we have $\left\langle t_{1} u, t_{1} u^{\prime}\right\rangle=\left\langle u, u^{\prime}\right\rangle$, and $t_{1}$ is orthogonal. Since $g$ satisfies $\mu(g)=\beta$, then $t_{2}=t_{1} g$ satisfies $\mu\left(t_{2}\right)=\beta$.

We now deal with the case that the minimal polynomial of $g$ is of the form $q(x)^{s}$, where $q(x)$ is irreducible and self- $\beta$-adjoint. It follows from [1, Lemmas 4 and 5] and the cyclic case above that we may assume that $V$ is the sum of two cyclic spaces, and further we may assume that for any $u_{1} \in V$ satisfying $q(g)^{s-1} u_{1} \neq 0$, the cyclic space $U_{1}$ generated by $u_{1}$ is degenerate, and for an appropriate $u_{2} \in V$, we have $V=U_{1} \oplus U_{2}$ where $U_{2}$ is the cyclic space generated by $u_{2}$. We follow the proof of [1, Proposition 3(iii)]. We may write $U_{1}=P_{1} \oplus Q_{1}$ where $P_{1}$ is spanned by vectors of the form $\left(g^{k}+\beta^{k} g^{-k}\right) u_{1}$ and $Q_{1}$ is spanned by vectors of the form $\left(g^{k}-\beta^{k} g^{-k}\right) u_{1}$.

There are two different cases, the first is when either $q(x)$ is relatively prime to $x^{2}-\beta$ or $q(x)=x^{2}-\beta$ and $s$ is odd. In this case, we find a $u_{2} \in Q_{1}^{\perp}$, and $V=U_{1} \oplus U_{2}$ where $U_{2}$ is cyclically generated by $u_{2}$. Then $U_{2}=P_{2} \oplus Q_{2}$, where $P_{2}$ is spanned by vectors of the form $\left(g^{k}+\beta^{k} g^{-k}\right) u_{2}$ and $Q_{2}$ is spanned by vectors of the form $\left(g^{k}-\beta^{k} g^{-k}\right) u_{2}$. Letting $P=P_{1} \oplus P_{2}$ and $Q=Q_{1} \oplus Q_{2}$, we are able to show that if $t_{1}$ is the involution with $P$ as its +1 eigenspace and $Q$ as its -1 eigenspace, then $\left(t_{1} g\right)^{2}=\beta I$ (for the symplectic group, we actually show this in the second case of Proposition 3(iii)). We need to show that $t_{1}$ is orthogonal. From the cyclic case above, we have $P_{i} \perp Q_{i}$ for $i=1,2$. In the proof of [1, Proposition 3(iii)], we show that $P_{i} \perp Q_{j}$ for $i \neq j$. So now $P \perp Q$, and from the argument in the cyclic case above, we have that $t_{1}$ is orthogonal, and so $t_{2}=t_{1} g$ satisfies $\mu\left(t_{2}\right)=\beta$.

In the case that $q(x)=x^{2}-\beta$ and $s$ is even, we are able to find a $u_{2} \in P_{1}^{\perp}$ such that $V=U_{1} \oplus U_{2}$, where $U_{2}$ is the space cyclically generated by $u_{2}$. We define $P_{2}$ and $Q_{2}$ as before. We let $P=P_{1} \oplus Q_{2}$ be the +1 eigenspace and $Q=P_{2} \oplus Q_{1}$ be the -1 eigenspace of an involution $t_{1}$, and this satisfies $\left(t_{1} g\right)^{2}=\beta I$. To show $t_{1}$ is orthogonal, we need only show that $P \perp Q$ and appeal to the cyclic case above. We have already shown $P_{i} \perp Q_{i}$ for $i=1,2$, so now we need $P_{1} \perp P_{2}$ and $Q_{1} \perp Q_{2}$. We have:

$$
\begin{aligned}
& \left\langle\left(g^{k} \pm \beta^{k} g^{-k}\right) u_{1},\left(g^{l} \pm \beta^{l} g^{-l}\right) u_{2}\right\rangle \\
& \quad=\left\langle\left(g^{k} \pm \beta^{k} g^{-k}\right) u_{1}, g^{l} u_{2}\right\rangle \pm\left\langle\left(g^{k} \pm \beta^{k} g^{-k}\right) u_{1}, \beta^{l} g^{-l} u_{2}\right\rangle \\
& \quad=\left\langle\beta^{l} g^{-l}\left(g^{k} \pm \beta^{k} g^{-k}\right) u_{1}, u_{2}\right\rangle \pm\left\langle g^{l}\left(g^{k} \pm \beta^{k} g^{-k}\right) u_{1}, u_{2}\right\rangle \\
& \quad= \pm\left\langle\left(g^{l} \pm \beta^{l} g^{-l}\right)\left(g^{k} \pm \beta^{k} g^{-k}\right) u_{1}, u_{2}\right\rangle \\
& \quad=0
\end{aligned}
$$

since

$$
\begin{aligned}
\left(g^{l}\right. & \left. \pm \beta^{l} g^{-l}\right)\left(g^{k} \pm \beta^{k} g^{-k}\right) u_{1} \\
& =\left(g^{l+k}+\beta^{l+k} g^{-(l+k)}\right) u_{1} \pm \beta^{k}\left(g^{l-k}+\beta^{l-k} g^{-(l-k)}\right) u_{1} \in P_{1}
\end{aligned}
$$


and $u_{2} \in P \stackrel{\perp}{\perp}$. So now as before, we have $t_{1}$ orthogonal. This exhausts all cases, and the theorem is proved.

Corollary 1 Any element of $g \in \mathrm{GO}(V)$ is conjugate to $\mu(g) g^{-1}$ by an orthogonal involution.

\section{Application over a finite field}

Let $G$ be a finite group with an order 2 automorphism $\iota$, let $(\pi, V)$ be an irreducible complex representation, and let $\hat{\pi}$ denote the contragredient representation. If ${ }^{\iota} \pi \cong \hat{\pi}$, where ${ }^{\imath} \pi(g)=\pi\left({ }^{\imath} g\right)$, then we obtain a bilinear form $B_{\iota}: V \times V \rightarrow \mathbb{C}$ satisfying

$$
B_{\iota}\left(\pi(g) v,{ }^{\prime} \pi(g) w\right)=B_{\iota}(v, w) \text { for every } v, w \in V .
$$

By Schur's Lemma, this bilinear form is unique up to scalar, which means we have, for all $v, w \in V$,

$$
B_{\iota}(v, w)=\varepsilon_{\iota}(\pi) B_{\iota}(w, v),
$$

where $\varepsilon_{\iota}(\pi)= \pm 1$. That is, $B_{\iota}$ is either symmetric or skew-symmetric. Since the character of $\hat{\pi}$ is $\bar{\chi}$ if $\chi$ is the character of $\pi$, then ${ }^{l} \pi \cong \hat{\pi}$ is equivalent to ${ }^{l} \chi=\bar{\chi}$.

Let $\mathbb{F}_{q}$ be the finite field of $q$ elements, and let $q$ be odd. We let $\mathrm{O}\left(n, \mathbb{F}_{q}\right)$ be the orthogonal group for any symmetric form (split or nonsplit) for an $\mathbb{F}_{q}$-vector space. Let $\operatorname{GO}\left(n, \mathbb{F}_{q}\right)$ be the corresponding orthogonal similitude group with similitude character $\mu$.

Proposition 1 Let $q$ be odd and $G=\mathrm{GO}\left(n, \mathbb{F}_{q}\right)$. Define $\iota$ to be the order 2 automorphism of $G$ that acts as ${ }^{\prime} g=\mu(g)^{-1} g$. Then every irreducible representation $\pi$ of $G$ satisfies ${ }^{\prime} \pi \cong \hat{\pi}$, that is, $\varepsilon_{l}(\pi)= \pm 1$.

Proof From Corollary 2, we have $g$ is conjugate to $\mu(g) g^{-1}$, and so $g^{-1}$ is always conjugate to ${ }^{\prime} g$. Thus every character satisfies ${ }^{\prime} \chi=\bar{\chi}$, and so for every $\pi$ we have $\varepsilon_{l}(\pi)= \pm 1$.

Gow [2] showed that for $q$ odd, every irreducible representation of $\mathrm{O}\left(n, \mathbb{F}_{q}\right)$ is self-dual and orthogonal. This corresponds to $\iota$ being the identity automorphism, and $\varepsilon_{l}(\pi)=\varepsilon(\pi)=1$. We are able to apply his result in order to obtain the following stronger version of Proposition 1.

Theorem 2 Let $q$ be odd and $G=\mathrm{GO}\left(n, \mathbb{F}_{q}\right)$. Define $\iota$ to be the order 2 automorphism of $G$ that acts as ${ }^{\prime} g=\mu(g)^{-1} g$. Then every irreducible representation $\pi$ of $G$ satisfies $\varepsilon_{l}(\pi)=1$.

Proof Since $\varepsilon_{\iota}(\pi)= \pm 1$ from Proposition 1, then we have a bilinear form $B_{\iota}$ as in (*).

Let $Z$ be the center of $G=\operatorname{GO}\left(n, \mathbb{F}_{q}\right)$ consisting of scalar matrices, and let $H=Z \cdot \mathrm{O}\left(n, \mathbb{F}_{q}\right)$. Then $H$ is an index 2 subgroup of $G$ consisting of elements whose similitude factor is a square in $\mathbb{F}_{q}^{\times}$. Every irreducible representation $\phi$ of $\mathrm{O}\left(n, \mathbb{F}_{q}\right)$ may be extended to an irreducible representation of $H$ by just extending the central character to $Z$, and so any irreducible representation of $H$ restricted to $\mathrm{O}\left(n, \mathbb{F}_{q}\right)$ 
is irreducible. Since $H$ is an index 2 subgroup of $G$, every irreducible representation $\pi$ of $G$ restricted to $H$ is either irreducible or the direct sum of 2 distinct irreducibles.

First assume that $(\pi, V)$ of $G$ restricts to an irreducible $\left(\pi^{\prime}, V\right)$ of $H$. Then $\pi^{\prime}$ restricted to $\mathrm{O}\left(n, \mathbb{F}_{q}\right)$ is some irreducible $\phi$. Note that for $g \in \mathrm{O}\left(n, \mathbb{F}_{q}\right)$, we have ${ }^{\prime} g=g$. Then for any $g \in \mathrm{O}\left(n, \mathbb{F}_{q}\right)$ and $u, v \in V$, we have

$$
B_{\iota}\left(\pi(g) u,{ }^{\prime} \pi(g) v\right)=B_{\iota}(\phi(g) u, \phi(g) v)=B_{\iota}(u, v)
$$

From Gow's result, we know that $\varepsilon(\phi)=1$, so there is a nondegenerate symmetric bilinear form, unique up to scalar, satisfying

$$
B(\phi(g) u, \phi(g) v)=B(u, v)
$$

for all $g \in \mathrm{O}\left(n, \mathbb{F}_{q}\right), u, v \in V$. So then $B_{\iota}$ must be a scalar multiple of $B$, and therefore must also be symmetric. Then we have $\varepsilon_{l}(\pi)=1$.

Now assume that the irreducible $(\pi, V)$ of $G$, when restricted to $H$, is isomorphic to the direct sum of two irreducible representations $\left(\pi_{1}, V_{1}\right)$ and $\left(\pi_{2}, V_{2}\right)$, which restrict to $\mathrm{O}\left(n, \mathbb{F}_{q}\right)$ to give the irreducibles $\left(\phi_{1}, V_{1}\right)$ and $\left(\phi_{2}, V_{2}\right)$, respectively. Now for any $g \in \mathrm{O}\left(n, \mathbb{F}_{q}\right)$, and $u, v \in V_{1}$, we have

$$
B_{\iota}\left(\phi_{1}(g) u, \phi_{1}(g) v\right)=B_{\iota}(u, v)
$$

Again from Gow's result, $\varepsilon\left(\phi_{1}\right)=1$, and so there is a symmetric nondegenerate $\mathrm{O}\left(n, \mathbb{F}_{q}\right)$-invariant bilinear form $B$ on $V_{1}$, unique up to scalar. Then if $B_{\iota}$ restricted to $V_{1} \times V_{1}$ is nondegenerate, it would have to be a scalar multiple of $B$, and so $B_{l}$ would be symmetric on $V_{1} \times V_{1}$. But since $B_{\iota}$ is either symmetric or skew-symmetric on all of $V \times V$, then being nondegenerate and symmetric on a subspace forces it to be symmetric everywhere. So now we must show $B_{\iota}$ is nondegenerate on $V_{1} \times V_{1}$.

For $g \in \mathrm{O}\left(n, \mathbb{F}_{q}\right), u \in V_{1}$, and $v \in V_{2}$, we have

$$
B_{\iota}\left(\pi(g) u,{ }^{\prime} \pi(g) v\right)=B_{\iota}\left(\phi_{1}(g) u, \phi_{2}(g) v\right)=B_{\iota}(u, v) .
$$

So if $B_{\iota}$ is nondegenerate on $V_{1} \times V_{2}$, then we would have $\hat{\phi}_{1} \cong \phi_{2}$. But $\phi_{2} \cong \hat{\phi}_{2}$, and so we would have $\phi_{2} \cong \phi_{1}$. This would imply that $\pi_{1} \cong \pi_{2}$, since the central characters of $\pi_{1}$ and $\pi_{2}$ agree with the central character of $\pi$. But we cannot have $\pi$ restricted to an index 2 subgroup be the direct sum of 2 isomorphic representations, by [5, Corollary 6.19]. So now $B_{\iota}$ must be zero on $V_{1} \times V_{2}$, by Schur's Lemma, which means $B_{\iota}$ must be nondegenerate on $V_{1} \times V_{1}$, since $B_{\iota}$ is nondegenerate on $V \times V$ and $V=V_{1} \oplus V_{2}$. Therefore, $B_{\iota}$ is symmetric, and $\varepsilon_{\iota}(\pi)=1$.

Kawanaka and Matsuyama [6] obtained a formula for the invariants $\varepsilon_{l}(\pi)$ which generalized the classical formula of Frobenius and Schur. One of the results in [6], which generalizes the Frobenius-Schur involution formula, is that if $\varepsilon_{\iota}(\pi)=1$ for all irreducible representations $\pi$ of a group $G$, then the sum of the degrees of the irreducibles of $G$ is equal to the number of elements in $G$ satisfying $g^{\prime} g=1$. From this and Theorem 2, we obtain the following. 
Corollary 2 Let $q$ be odd and let $G=\mathrm{GO}\left(n, \mathbb{F}_{q}\right)$. The sum of the degrees of the irreducible representations of $G$ is equal to

$$
\left|\left\{g \in G \mid g^{2}=\mu(g) I\right\}\right|
$$

It is perhaps worth noting that in the case of the group of similitudes for a split orthogonal group over $\mathbb{F}_{q}$, this is equal to the number of symmetric matrices in $G$.

\section{Acknowledgements}

The author thanks Dipendra Prasad for pointing out the importance and interest of the orthgonal case, and the referee for several helpful suggestions.

\section{References}

[1] Vinroot, C.R., 2004, A factorization in GSp(V). Linear and Multilinear Algebra, 52(6), 385-403.

[2] Gow, R., 1985, Real representations of the finite orthogonal and symplectic groups of odd characteristic. Journal of Algebra, 96(1), 249-274.

[3] Adler, J.D. and Prasad, D., On certain multiplicity one theorems. Israel Journal of Mathematics (To appear).

[4] Wonenburger, M., 1966, Transformations which are products of two involutions. Journal of Mathematics and Mechanics, 16, 327-338.

[5] Isaacs, I.M., 1976, Character theory of finite groups. In: Pure and Applied Mathematics, Vol. 69 (New York: Academic Press [Harcourt Brace Jovanovich Publishers]).

[6] Kawanaka, N. and Matsuyama, H., 1990, A twisted version of the Frobenius-Schur indicator and multiplicity-free representations. Hokkaido Mathematical Journal, 19(3), 495-508. 